Review

\title{
Selective Survey on Spaces of Closed Subgroups of Topological Groups
}

\section{Igor V. Protasov $\mathbb{B}$}

Faculty of Computer Science and Cybernetics, Kyiv University, Academic Glushkov pr. 4d, 03680 Kyiv, Ukraine; i.v.protasov@gmail.com

Received: 8 October 2018 ; Accepted: 24 October 2018 ; Published: 26 October 2018

check for updates

Abstract: We survey different topologizations of the set $\mathcal{S}(G)$ of closed subgroups of a topological group $G$ and demonstrate some applications using Topological Groups, Model Theory, Geometric Group Theory, and Topological Dynamics.

Keywords: space of closed subgroups; Chabauty topology; Vietoris topology; Bourbaki uniformity

MSC: 22A05; 22B05; 54B20; 54D30

For a topological group $G, \mathcal{S}(G)$ denotes the set of all closed subgroups of $G$. There are many ways to endow $\mathcal{S}(G)$ with a topology related to the topology of $G$. Among these methods, the most intensively studied are the Chabauty topology, rooted in Geometry of Numbers, and the Vietoris topology, based on General Topology; both coincide if $G$ is compact. The spaces of closed subgroups are interesting by their own merits, but they also have some deep applications in Topological Groups and Model Theory, Geometric Group Theory, and Dynamical Systems. This survey is my subjective look at this area.

\section{Chabauty Spaces}

\subsection{From Minkowski to Chabauty}

We recall that a lattice $L$ in $\mathbb{R}^{n}$ is a discrete subgroup of rank $n$. We define $\min L$ as the length of the shortest nonzero vector from $L$, and we define $v o l\left(\mathbb{R}^{n} / L\right)$ as the volume of a basic parallelepiped of $L$.

A sequence $\left(L_{m}\right)_{m \in \omega}$ of lattices in $\mathbb{R}^{n}$ converges to the lattice $L$ if, for each $m \in \omega$, one can choose a basis $a_{1}(m), \ldots, a_{n}(m)$ of $L_{m}$ and a basis $a_{1}, \ldots, a_{n}$ of $L$ such that the sequence $\left(a_{i}(m)\right)_{m \in \omega}$ converges to $a_{i}$ for each $i \in\{1, \ldots, n\}$. This convergence of lattices was introduced by H. Minkowski [1], and its usage in Geometry of Numbers (see [2]) is based on the following theorem from K. Mahler [3].

Theorem 1. Let $\mathcal{M}$ be a set of lattices in $\mathbb{R}^{n}$. Every sequence in $\mathcal{M}$ has a convergent subsequence if and only if there exist two constants, $C>0$ and $c>0$, such that $\min L>c$, vol $\left(\mathbb{R}^{n} \backslash L\right)<C$ for each $L \in \mathcal{M}$.

What we know now is that Chabauty topology was invented by C. Chabauty in [4] in order to extend Theorem 1 to lattices in connected Lie groups.A discrete subgroup $L$ of a connected Lie group $G$ is called a lattice if the quotient space $G / L$ is compact.

Let $X$ be a Hausdorff locally compact space, and let $\exp X$ denote the set of all closed subsets of $X$. The sets

$$
\{F \in \exp X: F \cap K=\varnothing\},\{F \in \exp X: F \cap U \neq \varnothing\},
$$


where $K$ runs over all compact subsets of $X$ and $U$ runs over all open subsets of $X$, form the subbase of the Chabauty topology on exp X. The space exp X is compact and Hausdorff. If $X$ is discrete, then $\exp X$ is homeomorphic to the Cantor cube $\{0,1\}^{|X|}$.

We note also that a net $\left(F_{\alpha}\right)_{\alpha \in \mathcal{I}}$ converges in $\exp X$ to $F$ if and only if

- for every compact $K$ of $X$ such that $K \cap F=\varnothing$, there exists $\beta \in \mathcal{I}$ such that $F_{\alpha} \cap K=\varnothing$ for each $\alpha>\beta$

- $\quad$ for every $x \in F$ and every neighborhood $U$ of $x$, there exists $\gamma \in \mathcal{I}$ such that $F_{\alpha} \cap U \neq \varnothing$ for each $\alpha>\gamma$

If $G$ is a locally compact group, then $\mathcal{S}(G)$ is a closed subspace of $\exp G$ (so, $\mathcal{S}(G)$ is compact); $\mathcal{S}(G)$ is called the Chabauty space of $G$.

Theorem 2. [4]. Let $G$ be a connected unimodular Lie group. A set $\mathcal{M}$ of lattices in $G$ is relatively compact in $\mathcal{M}$ if and only if there exists a constant $C>0$ and a neighborhood $U$ with the identity e of $G$ such that $L \cap U=\{e\}$ and vol $(G / L)<C$ for each $L \in \mathcal{M}$.

There was some technical improvement made in [5] and the paper [4], which is included in [6], Chapter 8.

\subsection{Pontryagin-Chabauty Duality}

This duality was established in [7] and detailed in [8]. We use the standard abbreviation LCA for a locally compact Abelian group. Let $G$ be an LCA-group $G^{\wedge}$, let denote its dual group $G^{\wedge}=$ $\operatorname{Hom}(G, \mathbb{R} / \mathbb{Z})$, and let $\varphi$ denote the canonical bijection $\mathcal{S}(G) \longrightarrow \mathcal{S}\left(G^{\wedge}\right), \varphi(X)=\left\{f \in G^{\wedge}: X \subseteq\right.$ ker $\left.f\right\}$.

Theorem 3. For every $L C A$-group $G$, the bijection $\varphi: \mathcal{S}(G) \longrightarrow \mathcal{S}\left(G^{\wedge}\right)$ is a homeomorphism.

Typically, Theorem 3 is applied to replace $\mathcal{S}(G)$ by $\mathcal{S}\left(G^{\wedge}\right)$ in the case of a compact Abelian group $G$.

In what follows, we use the following notations: $\mathbb{C}_{n}$ is a cyclic group of order $n, \mathbb{C}_{p^{\infty}}$ is a quasi-cyclic (or Prüfer) $p$-group, $\mathbb{Z}$ is a discrete group of integers, $\mathbb{Z}_{p}$ is the group of $p$-adic integers, and $\mathbb{Q}_{p}$ is an additive group of a field of $p$-adic numbers.

\section{3. $S(G)$ for Compact $G$}

The following two lemmas from [9] are the basic technical tools in this area.

Lemma 1. If $G, H$ are compact groups and $\varphi: G \longrightarrow H$ is a continuous surjective homomorphism, then the mapping $\mathcal{S}(G) \longrightarrow \mathcal{S}(H), X \longmapsto \varphi(X)$ is continuous and open.

The continuity is easily deduced, but to prove the openness, we need

Lemma 2. Let $G$ be a compact group, $X \in \mathcal{S}(G)$. Then, the following subsets form a base of the neighborhoods of $X$ is $\mathcal{S}(G)$ :

$$
\mathcal{N}_{X}\left(U, N, x_{1}, \ldots, x_{n}\right)=\left\{u^{-1} Y u: u \in U, Y \in \mathcal{S}(G), Y \subseteq X N, Y \cap x_{1} U \neq \varnothing, \ldots, Y \cap x_{n} U \neq \varnothing,\right\}
$$

where $U$ is a neighborhood of the identity of $G, N$ is closed normal subgroup such that $G / N$ is a Lie group, and $x_{1}, \ldots, x_{n}$ are arbitrary elements of $X, n \in \mathbb{N}$. 
In particular, if $G$ is a compact Lie group, then Lemma 2 states that there is a neighborhood $\mathcal{N}$ of $X$ such that each subgroup $Y \in \mathcal{N}$ is conjugated to some subgroup of $X$. The Montgomery-Yang theorem on tubes [10] (see also ([11], Theorem 5.4, Chapter 2)) plays the key role in the proof of Lemma 2.

We recall that the cellularity (or Souslin number) $c(X)$ of a topological space $X$ is the supremum of cardinalities of disjoint families of open subsets of $X$. A topological space $X$ is called dyadic if $X$ is a continuous image of some Cantor cube $\{0,1\}^{\kappa}$.

The weight $w(X)$ of a topological space $X$ is the minimal cardinality of the open bases of $X$.

Theorem 4. [9]. For every compact group $G$, we have $c(\mathcal{S}(G)) \leq \aleph_{0}$. In addition, if $w(G) \leq \aleph_{1}$, then $\mathcal{S}(G)$ is dyadic.

Theorem 5. [12]. Let a group $G$ be either profinite or compact and Abelian. If $w(G)>\aleph_{2}$, then the space $\mathcal{S}(G)$ is not dyadic.

Theorem 6. [12]. Let $G$ be an infinite compact Abelian group such that $w(G) \leq \aleph_{1}$. Then, the space $\mathcal{S}(G)$ is homeomorphic to the Cantor cube $\{0,1\}^{w(G)}$ if and only if $\mathcal{S}(G)$ has no isolated points.

An Abelian group $G$ is called Artinian if every increasing chain of subgroups of $G$ is finite; every such group is isomorphic to the direct sum $\oplus_{p \in F} \mathbb{C}_{p^{\infty}} \oplus K$, where $F$ is a finite set of primes, and $K$ is a finite subgroup. An Abelian group $G$ is called minimax if $G$ has a finitely generated subgroup $N$ such that $G / N$ is Artinian.

Theorem 7. [12]. For a compact Abelian group $G$, the space $\mathcal{S}(G)$ has an isolated point if and only if the dual group $G^{\wedge}$ is minimax.

\section{4. $S(G)$ for $L C A G$}

The space $\mathcal{S}(\mathbb{R})$ is homeomorphic to the segment [0,1]. By [13], $\mathcal{S}\left(\mathbb{R}^{2}\right)$ is homeomorphic to the sphere $\mathbf{S}^{4}$. For $n \geq 3, \mathcal{S}\left(\mathbb{R}^{n}\right)$ is not a topological manifold and its structure is far from understood (see [14]).

Theorem 8. [15]. The space $\mathcal{S}(G)$ of an $L C A$-group $G$ is connected if and only if $G$ has a subgroup topologically isomorphic to $\mathbb{R}$.

If $F$ is a non-solvable finite group, then $\mathcal{S}(\mathbb{R} \times F)$ is not connected ([8], Proposition 8.6).

Theorem 9. [8]. The space $\mathcal{S}(G)$ of an $L C A$-group $G$ is totally disconnected if and only if $G$ is either totally disconnected or each element of $G$ belongs to a compact subgroup.

Some more information on $\mathcal{S}(G)$ for LCA $G$ can be found in [8] and the references therein, particularly on the topological dimension of $\mathcal{S}(G)$.

By Theorems 3 and $4, c(\mathcal{S}(G)) \leq \aleph_{0}$ for every discrete Abelian group. We say that a topological space $X$ has the Shanin number $\omega$ if any uncountable family $\mathcal{F}$ of the non-empty open subsets of $X$ has an uncountable subfamily $\mathcal{F}^{\prime}$ such that $\cap \mathcal{F}^{\prime} \neq \varnothing$. Evidently, if a space $X$ has the Shanin number $\omega$, then $c(X) \leq \aleph_{0}$. By [16], Theorem 1, for every discrete Abelian group $G$, the space $\mathcal{S}(G)$ has the Shanin number $\omega$. By [16], Theorem 3, for every infinite cardinal $\tau$, there exists a solvable discrete group $G$ such that $c(\mathcal{S}(G))=|G|=\tau$. 


\section{5. $S(G)$ as a Lattice}

The set $S(G)$ has the natural structure of a lattice with the operations $\vee$ and $\wedge$, where $A \wedge B=A \cap B$, and $A \vee B$ is the smallest closed subgroup of $G$ containing $A$ and $B$. In this subsection, we formulate some results from [17] about the interrelations between the topological and lattice structures on $S(G)$.

For $g \in G, \overline{\langle g\rangle}$ denotes the subgroup of $G$ topologically generated by $g$. A totally disconnected locally compact group $G$ is called periodic if $\langle g\rangle$ is compact for each $g \in G$. In this case, $\pi(G)$ denotes the set of all prime numbers such that $p \in \pi(G)$ if and only if $g \in G$ such that $\overline{\langle g}\rangle$ is topologically isomorphic either to $\mathbb{C}_{p^{n}}$ or to $\mathbb{Z}_{p}$; this $g$ is called a topological p-element.

Theorem 10. For a compact group $G$, the following statements are equivalent:

(i) $\wedge$ is continuous;

(ii) $\wedge$ and $\vee$ are continuous;

(iii) $G$ is the semidirect product $K \lambda P$, where $K$ is profinite with finite Sylow $p$-subgroups, $P$ is Abelian profinite and each Sylow $p$-subgroup of $G$ is $\mathbb{Z}_{p}, \pi(K) \cap \pi(P)=\varnothing$, and the centralizer of each Sylow -subgroup of $G$ has a finite index in $G$.

Theorem 11. For a locally compact group $G$, the operation $\wedge$ is continuous if and only if the following conditions are satisfied:

(i) $G$ is either discrete or periodic;

(ii) $\wedge$ is continuous in $\mathcal{S}(H)$ for each compact subgroup $H$ of $G$;

(iii) the centralizer of each topological p-element of $G$ is open.

We recall that a torsion group $G$ is layer-finite if the set $\left\{g \in G: g^{n}=e\right\}$ is finite for each $n \in \mathbb{N}$. A layer-finite group $G$ is called thin if each Sylow $p$-subgroup of $G$ is finite (equivalently, $G$ has no subgroup isomorphic to $\mathbb{C}_{p^{\infty}}$ ).

Theorem 12. Let $G$ be a locally compact group. The operations $\wedge$ and $\vee$ are continuous if and only if $G$ is periodic and topologically isomorphic to $A \times B \times(C \lambda D)$, where $C$ has a dense thin layer-finite subgroup; $A, B, D$ are Abelian with Sylow p-subgroups $\mathbb{C}_{p^{\infty}}, \mathbb{Q}_{p}$, or $\mathbb{Z}_{p}$; the sets $\pi(A), \pi(B), \pi(G), \pi(D)$ are pairwise disjoint; and the centralizer of each Sylow p-subgroup of $G$ is open.

\subsection{From Chabauty to Local Method}

A topological group $G$ is called topologically simple if each closed normal subgroup of $G$ is either $G$ or $\{e\}$. Every topologically simple LCA-group is discrete, and either $G=\{e\}$ or $G$ is isomorphic to $\mathbb{C}_{p}$.

Following the algebraic tradition, we say that a group $G$ is locally nilpotent (solvable) if every finitely generated subgroup is nilpotent (solvable).

In [18], Problem 1.76, V. Platonov asked whether there exists a non-Abelian, topologically simple, locally compact, locally nilpotent group. Here, we present the negative answer to this question for the locally solvable group obtained in [19].

Let $G$ be a locally compact, locally solvable group. We take $g \in G \backslash\{e\}$, choose a compact neighborhood $U$ of $G$, and denote by $\mathcal{F}$ the family of all topologically finitely generated subgroups of $G$ containing $g$. We may assume that $G$ is not topologically finitely generated, so $\mathcal{F}$ is directed by the inclusion $\subset$. For each $F \in \mathcal{F}$, we choose $A_{F}, B_{F} \in \mathcal{S}(F)$ such that $B_{F} \subset A_{F} ; A_{F}$ and $B_{F}$ are normal in $F$, $A_{F} \cap U \neq \varnothing, B_{F} \cap U=\varnothing$, and $A_{F} / B_{F}$ is Abelian. Since $\mathcal{S}(G)$ is compact, we can choose two subnets $\left(A_{\alpha}\right)_{\alpha \in \mathcal{I}},\left(B_{\alpha}\right)_{\alpha \in \mathcal{I}}$ of the nets $\left(A_{F}\right)_{F \in \mathcal{F}},\left(B_{F}\right)_{F \in \mathcal{I}}$ which converge to $A, B \in \mathcal{S}(G)$. Then $A, B$ are normal 
in $G$, and $A / B$ is Abelian. Moreover, $x \notin B$ and $A \cap U \neq \varnothing$. If $A \neq\{G\}$, then $A$ is a proper normal subgroup of $G$; otherwise, $G / B$ is Abelian.

In [20], the Chabauty topology was defined on some systems of closed subgroups of a locally compact group $G$. A system $\mathfrak{A}$ of closed subgroups of $G$ is called subnormal if

- $\quad \mathfrak{A}$ contains $\{e\}$ and $G$;

- $\mathfrak{A}$ is linearly ordered by the inclusion $\subset$;

- for any subset $\mathfrak{M}$ of $\mathfrak{A}$, the closure of $\bigcup_{F \in \mathfrak{M}} F \in \mathfrak{A}$ and $\bigcap_{F \in \mathfrak{M}} F \in \mathfrak{A}$;

- whenever $A$ and $B$ comprise a jump in $\mathfrak{A}$ (i.e., $B \subset A$, and no members of $\mathfrak{A}$ lie between $B$ and $A$ ), $B$ is a normal subgroup of $A$.

If the subgroups $A, B$ form a jump, then $A / B$ is called a factor of $G$. The system is called normal if each $A \in \mathfrak{A}$ is normal in $G$.

A group $G$ is called an RN-group if $G$ has a normal system with Abelian factors. Among the local theorems from [20], one can find the following: if every topologically finitely generated subgroup of a locally compact group $G$ is an $R N$-group, then $G$ is an $R N$-group. In particular, every locally compact, locally solvable group is an $\mathrm{RN}$-group.

In 1941 (see ([21], pp. 78-83), A.I. Mal'tsev obtained local theorems for discrete groups as applications of the following general local theorem: if every finitely generated subsystem of an algebraic system $A$ satisfies some property $\mathcal{P}$, which can be defined by some quasi-universal second-order formula, then $A$ satisfies $\mathcal{P}$.

In [22], Mal'tsev's local theorem was generalized on a topological algebraic system. The part of the model-theoretical Compactness Theorem in Mal'tsev's arguments employs some convergents of closed subsets. A net $\left(F_{\alpha}\right)_{\alpha \in \mathcal{I}}$ of closed subsets of a topological space $X S$-converges to a closed subset $F$ if

- for every $x \in F$ and every neighborhood $U$ of $x$, there exists $\beta \in \mathcal{I}$ such that $F_{\alpha} \cap U \neq \varnothing$ for each $\alpha>\beta$;

- for every $y \in X \backslash F$, there exist a neighborhood $\mathcal{V}$ of $y$ and a $\gamma \in \mathcal{I}$ such that $F_{\alpha} \cap \mathcal{V}=\varnothing$ for each $\alpha>\gamma$.

Every net of closed subsets of an arbitrary (!) topological space has a convergent subnet. If $X$ is a Hausdorff locally compact space, then the $S$-convergence coincides with the convergence in the Chabauty topology.

\subsection{Spaces of Marked Groups}

Let $F_{k}$ be the free group of rank $k$, with the free generators $x_{1}, \ldots, x_{k}$, and let $\mathcal{G}_{k}$ denote the set of all normal subgroups of $F_{k}$. In the metric form, the Chabauty topology on $\mathcal{G}_{k}$ was introduced in [23] as a reply to Gromov's idea of the topologizations of some sets of groups [24].

Let $G$ be a group generated by $g_{1}, \ldots, g_{k}$. The bijection $x_{i} \longmapsto g_{i} g_{1}, \ldots, g_{n}$ can be extended to the homomorphism $f: F_{k} \longrightarrow G$. With the correspondence $G \longmapsto \operatorname{ker} f, \mathcal{G}_{k}$ is called the space marked k-generated groups.

A couple of papers in development by [23] are aimed at understanding how large, in the topological sense, are the well-known classes of finitely generated groups, or how a given $k$-generated group is placed in $\mathcal{G}_{k}$ (see [25]). Among the applications of $\mathcal{G}_{k}$, we mention the construction of topologizable Tarski Monsters in [26]. 


\subsection{Dynamical Development}

Every locally compact group $G$ acts on the Chabauty space $\mathcal{S}(G)$ by the rule: $(g, H) \longmapsto g^{-1} H g$. Under this action, every minimal closed invariant subset of $\mathcal{S}(G)$ is called a uniformly recurrent subgroup (URS). The study of URSs was initiated by Glasner and Weiss [27] with the following observation.

Let $G$ be a locally compact group $G$ acting on a compact $X$ so that is $G$ minimal, i.e., the orbit of each point $x \in X$ is dense. We consider the mapping Stab $: X \longrightarrow \mathcal{S}(G)$, defined by $\operatorname{Stab}(x)=\{g \in G: g x=x\}$. Then, there is the unique URS contained in the closure of Stab(X). This URS is called the stabilizer URS. Glasner and Weiss asked whether every URS of a locally compact group $G$ arises as the stabilizer URS of a minimal action of $G$ on a compact space. This question was answered in the affirmative in [28].

\section{Vietoris Spaces}

For a topological space $X$, the Vietoris topology on the set $\exp X$ of all closed subsets of $X$ is defined by the subbase of the open sets

$$
\{F \in \exp X: F \subseteq U\},\{F \in \exp X: F \cap V \neq \varnothing\},
$$

where $U, \mathcal{V}$ run over all open subsets of $X$.

A net $\left(F_{\alpha}\right)_{\alpha \in \mathcal{I}}$ converges to $F$ in $\exp X$ if and only if

- for each open subset $U$ of $X$ such that $F \subseteq U$, there exists $\beta \in \mathcal{I}$ such that $F_{\alpha} \subseteq U$ for each $\alpha>\beta$;

- for each $x \in F$ and each neighborhood $\mathcal{V}$ of $x$, there exists $\gamma \in \mathcal{I}$ such that $F_{\alpha} \cap \mathcal{V} \neq \varnothing$ for each $\alpha>\gamma$.

If $X$ is regular, then $\mathcal{S}(G)$ is closed in $\exp G$. To my knowledge, the spaces $\mathcal{S}(G)$, where $G$ needs not be compact, endowed with Vietoris topologies appeared in [29] with the characterization of LCA-groups $G$ such that the canonical mapping $\varphi: \mathcal{S}(G) \longrightarrow \mathcal{S}\left(G^{\wedge}\right)$ is a homeomorphism.

\subsection{Compactness}

We cannot ask for a constructive description of arbitrary topological groups $G$ with compact space $\mathcal{S}(G)$ because we know nothing about $G$ with $S(G)=2$.

Theorem 13. [30]. Let $G$ be a locally compact group. The space $\mathcal{S}(G)$ is compact if and only if $G$ is one of the following groups:

(i) G is compact;

(ii) $\mathbb{C}_{p_{1}^{\infty}} \times \ldots \times \mathbb{C}_{p_{n}^{\infty}} \times K$, where $p_{1}, \ldots, p_{n}$ are distinct prime numbers, $K$ is finite, and each $p_{i}$ is not a divisor of $|K|$;

(iii) $Q_{p} \times K$, where $K$ is finite and $p$ does not divide $|K|$.

A similar characterization of groups with compact $\mathcal{S}(G)$ is given in [31], provided that $G$ has a base of neighborhoods at the identity consisting of subgroups.

Theorem 14. [32]. Let $G$ be a locally compact group. A closed subset $\mathcal{F}$ of $\mathcal{S}(G)$ is compact if and only if the following conditions are satisfied:

(i) every descending chain of non-compact subgroups from $F$ is finite; 
(ii) every closed subset $\mathcal{F}^{\prime}$ of $\mathcal{F}$ has only a finite number of non-compact subgroups maximal in $\mathcal{F}$;

(iii) if a closed subset $\mathcal{F}^{\prime}$ of $\mathcal{F}$ has no non-compact subgroups, then $\cup \mathcal{F}^{\prime}$ is compact.

Two corollaries: Every compact subset of $\mathcal{S}(G)$ consisting of non-compact subgroups is scattered; a subset $\mathcal{F}$ is compact if and only if $\mathcal{F}$ is countably compact.

For locally compact groups with the $\sigma$-compact space $\mathcal{S}(G)$ (see [33]), a description of the LCA-groups with locally compact space $\mathcal{S}(G)$ can be obtained in [34].

A topological group $G$ is called inductively compact if every finite subset of $G$ is contained in a compact subgroup. For a group $G, K(G)$ and $I K(G)$ denote the sets of all compact and closed inductively compact subgroups.

Theorem 15. [35]. For every locally compact group $G, I K(G)$ is the closure of $K(G)$.

Two corollaries: If $G$ is a connected Lie group, then $K(G)$ is closed; $\mathcal{S}(G)$ is a $k$-space for each locally compact group $G$ of countable weight, i.e., the topology of $\mathcal{S}(G)$ is uniquely determined by the family of all compact subsets of $\mathcal{S}(G)$.

\subsection{Metrizability and Normality}

LCA-groups $G$ with metrizable and normal space $\mathcal{S}(G)$ were characterized by S. Panasyuk in the candidate thesis Normality and metrizability of the space of closed subgroups, Kyiv University, 1989.

Theorem 16. For a discrete Abelian group $G$, the following statements are equivalent:

(i) $\mathcal{S}(G)$ is metrizable;

(ii) $\mathcal{S}(G)$ is normal;

(iii) $G$ has a finitely generated subgroup $H$ such that $G / H=\mathbb{C}_{p_{1}^{\infty}} \times \ldots \times \mathbb{C}_{p_{n}^{\infty}}$, where $p_{1}, \ldots, p_{n}$ are distinct primes.

In the general case, metrizability and normality of $\mathcal{S}(G)$ are not equivalent, but if $G$ is a connected semisimple Lie group, then $\mathcal{S}(G)$ is metrizable if and only if $\mathcal{S}(G)$ is normal if and only if $G$ is compact (see [36,37]). The space $\mathcal{S}(G)$ for every connected solvable Lie group is metrizable [36].

\subsection{Some Cardinal Invariants}

We remind the reader that $c(X)$ denotes the cellularity of $X$.

Theorem 17. [9]. For every infinite locally compact group $G$, we have $c(\mathcal{S}(G)) \leq c(G)$.

Theorem 18. [38]. For every locally compact group $G$, the following conditions are equivalent:

(i) $\mathcal{S}(G)$ is of countable pseudocharacter;

(ii) $\mathcal{S}(G)$ is of countable tightness;

(iii) $\mathcal{S}(G)$ is sequential;

(iv) $w(G) \leq \aleph_{0}$. 


\section{Other Topologizations}

\subsection{Bourbaki Uniformities}

Let $(X, \mathcal{U})$ be a uniform space. The uniformity $\mathcal{U}$ induces the uniformity $\widetilde{\mathcal{U}}$ on the set $\mathcal{F}(X)$ of all non-empty closed subsets of $X$ which have as a base the family of sets of the form

$$
\{(A, B) \in \mathcal{F}(X) \times \mathcal{F}(X): B \subseteq U(A), A \subseteq U(B)\}
$$

whenever $U \in \mathcal{U}$. The uniformity $\widetilde{\mathcal{U}}$ was introduced in [39] (Chapter $2, \S 1$ ), and $\widetilde{\mathcal{U}}$ is called the Bourbaki (sometimes, Hausdorff-Bourbaki) uniformity.

Let $G$ be a topological group. We endow $G$ with the left uniformity $L$ and $F(G)$ with the Bourbaki uniformity $\widetilde{L}$. We denote by $\mathcal{L}(G)$ and $\mathcal{B}(G)$ the subspaces of $\mathcal{F}(G)$ consisting of all subgroups and all totally bounded subsets of $G$.

Theorem 19. [40]. Let $G$ be a group with a base at the identity consisting of subgroups. The space $\mathcal{L}(G)$ is compact if and only if $G$ is totally bounded and $K \cap G$ is dense in $K$ for each closed subgroup $K$ from the completion of $G$.

In particular, if $\mathcal{L}(G)$ is compact, then $G$ is totally minimal.

Theorem 20. [40]. If a group $G$ is complete in the left uniformity, then $\mathcal{B}(G)$ is complete.

We recall that a topological group $G$ is almost metrizable if each neighborhood of $e$ contains a compact subgroup $K$ such that the set of all open subsets containing $K$ have a countable base. Every metrizable and every locally compact topological group is almost metrizable.

Theorem 21. [40]. If an almost metrizable group $G$ is complete in the left uniformity, then $\mathcal{F}(G)$ is complete.

In [41], Theorem 21 is proved with the bilateral uniformity on $G$ (and so on $\mathcal{F}(G)$ ) in place of the left uniformity.

\subsection{Functionally Balanced Groups}

For a topological group $G$, the set $\mathcal{F}(G)$ has the natural structure of a semigroup with the operation $(A, B) \longmapsto c l A B$.

Theorem 22. [42]. For a topological group $G$, the following statements are equivalent:

(i) $\mathcal{F}(G)$ is a topological semigroup;

(ii) for every subset $X$ of $G$ and every neighborhood $U$ of $e$, there exists a neighborhood $V$ of $e$ such that $V X \subseteq X U$;

(iii) every bounded left uniformly continuous function on $G$ is right uniformly continuous.

A topological group $G$ is called balanced (or a SIN-group) if the left and right uniformities of $G$ coincide. A group $G$ is called functionally balanced if $G$ satisfies (iii) of Theorem 22. The study of functionally balanced groups was initiated by G. Itzkowitz [43]. 
The equivalence of (ii) and (iii) in Theorem 22 is a criterion for a topological group to be functionally balanced. In [44], this criterion was used to show that each almost-metrizable functionally balanced group is balanced.

\subsection{Lattice Topologies}

These topologies on a complete lattice $\mathcal{L}(G)$ of closed subgroups are algebraically defined by the lattice structure of $\mathcal{L}(G)$.

For example, a net $\left(A_{\alpha}\right)_{\alpha \in \mathcal{I}}$ in $\mathcal{L}(G)$ order-converges to $A \in \mathcal{L}(G)$ if there exist two nets $\left(B_{\alpha}\right)_{\alpha \in \mathcal{I}}$, $\left(C_{\alpha}\right)_{\alpha \in \mathcal{I}}$ in $\mathcal{L}(G)$ such that, for each $\alpha \in \mathcal{I}, B_{\alpha} \subseteq A_{\alpha} \subseteq C_{\alpha}$ and $\vee_{\alpha \in \mathcal{I}} B_{\alpha}=\wedge_{\alpha \in \mathcal{I}} C_{\alpha}=A$. By [45], for a compact group $G$, every net in $\mathcal{L}(G)$ has an order-convergent subset if and only if $\mathcal{L}(G)$ endowed with the Shabauty topology is a topological lattice (see Theorem 10).

More on the lattices' topologies on $\mathcal{L}(G)$ in the case of a compact $G$ can be found in [46].

\subsection{Segment Topologies}

Let $G$ be a topological group; $\mathcal{P}_{G}$ is the family of all subsets of $G$, and $[G]^{<\omega}$ is the family of all finite subsets of $G$. Each pair $\mathcal{A}, \mathcal{B}$ of subsets of $\mathcal{P}_{G}$ closed under finite unions defines the segment topology on $\mathcal{L}(G)$ with a base consisting of the segments

$$
[A, G \backslash B]=\{X \in \mathcal{L}(G): A \subseteq X \subseteq G \backslash B\}, A \in \mathcal{A}, B \in \mathcal{B} .
$$

These topologies are described in [47] in the following three cases: $\mathcal{A}=\mathcal{B}=[G]^{<\omega} ; \mathcal{A}=\mathcal{P}_{G}$ and $\mathcal{B}=[G]^{<\omega} ; \mathcal{A}=[G]^{<\omega}, \mathcal{B}=\mathcal{P}_{G}$

\section{5. $(\Sigma, \Theta)$-Topologies}

This general construction for topologizations of the set $\mathcal{L}(G)$ of closed subgroups of a topological group $G$ from [48] produces Chabauty, Vietoris, and Bourbaki topologies, along with plenty of other topologies.

We assume that, for each $H \in \mathcal{L}(G), \Sigma(H)$ is some family of open subsets of $G, \Sigma=\cup_{H \in \mathcal{L}(G)} \Sigma(H)$, and the following conditions are satisfied:

- if $U, \mathcal{V} \in \Sigma(H)$, then $U \cap \mathcal{V}$ contains some $W \in \Sigma(H)$;

- for every $U \in \Sigma(H)$, there exists $\mathcal{V} \in \Sigma(H)$ such that $U \in \Sigma(K)$ for each $K \in \mathcal{L}(G), K \subseteq \mathcal{V}$;

- $\bigcap_{U \in \Sigma(H)} \bar{U}=H$ for each $H \in \mathcal{L}(G)$.

Then, the family $\{X \in \mathcal{L}(G): X \subseteq U\}, U \in \Sigma$, is a base for the $\Sigma$-topology on $\mathcal{L}(G)$.

Let $\tau$ denote the topology of $G$, and let $\mathcal{P}_{\tau}$ denote the family of all subsets of $\tau$. We assume that, for each $H \in \mathcal{L}(G), \Theta(H)$ is some subset of $\mathcal{P}_{\tau}$ such that the following conditions are satisfied:

- for every $\alpha, \beta \in \Theta(H)$, there is a $\gamma \in \Theta(H)$ such that $\alpha<\gamma, \beta<\gamma(\alpha<\beta$ means that, for every $U \in \alpha$, there exists $V \in \beta$ such that $V \subseteq U$ );

- for every $\alpha \in \Theta(H)$, there exists $\beta \in \Theta(H)$ such that if $K \in \mathcal{L}(G)$ and $K \cap V \neq \varnothing$ for each $V \in \beta$, then $\alpha<\gamma$ for some $\gamma \in \Theta(K)$;

- for each $H \in \mathcal{L}(G)$ and every neighborhood $V$ of $x$, there exists $\alpha \in \Theta(H)$ such that $x \in U, U \subseteq V$ for some $U \in \alpha$.

Then, the family $\{X \in \mathcal{L}(G): X \cap U \neq \varnothing$ for each $U \in \alpha\}$, where $\alpha \in \Theta(H), H \in \mathcal{L}(G)$, is a base for the $\Theta$-topology on $\mathcal{L}(G)$. 
The upper bound of $\Sigma$ - and $\Theta$-topologies is called the $(\Sigma, \Theta)$-topology. A net $\left(H_{\alpha}\right)_{\alpha \in \mathcal{I}}$ converges in $(\Sigma, \Theta)$-topology to $H \in \mathcal{L}(G)$ if and only if

- for any $U \in \Sigma(H)$, there exists $\beta \in \mathcal{I}$ such that $H_{\alpha} \subseteq U$ for each $\alpha>\beta$;

- for any $\alpha \in \Theta(H)$, there exists $\gamma \in \mathcal{I}$ such that $H_{\alpha} \cap \mathcal{V} \neq \varnothing$ for each $\alpha>\gamma$.

In [48], one can find characterizations of $G$ with a compact and discrete $\mathcal{L}(G)$ for some concrete $(\Sigma, \Theta)$-topologies.

\subsection{Hyperballeans of Groups}

Let $G$ be a discrete group. The set $\left\{F g: g \in G, F \in[G]^{<\omega}\right\}$ is a family of balls in the finitary coarse structure on $G$. For definitions of coarse structures and balleans, see $[49,50]$. The finitary coarse structure on $G$ induces the coarse structure on $\mathcal{L}(G)$ in which $\{X \in \mathcal{L}(G): X \subseteq F A, A \in F X\}, F \in[G]^{<\omega}$ is the family of balls centered at $A \in \mathcal{L}(G)$. The set $\mathcal{L}(G)$ endowed with the finitary coarse structure is called a hyperballean of $G$. Hyperballeans of groups, carefully studied in [51], can be considered as asymptotic counterparts of Bourbaki uniformities.

Funding: This research received no external funding.

Conflicts of Interest: The author declares no conflicts of interest.

\section{References}

1. Minkowski, H. Geometrie der Zahlen; R.G. Teubner: Leipzig/Berlin, Germany, 1910.

2. Cassels, J.W.S. An Introduction to the Geometry of Nombres; Classics in Mathematics; Springer: Berlin, Germany, 1997.

3. Mahler, K. On lattice points in n-dimensional star bodies: I. Existence theorems. Proc. R. Soc. Lond. 1946, 187, 151-187. [CrossRef]

4. Chabauty, C. Limite d'ensembles et geometrie des nombres. Bull. Soc. Math. Fr. 1950, 78, 143-151. [CrossRef]

5. Macbeath, A.M.; Swierczkowski, S. Limits of lattices in a compactly generated group. Can. J. Math. 1960, 12, 427-437. [CrossRef]

6. Bourbaki, N. Éléments de Mathématique. Fascicule XXIX. Livre VI: Intégration. Chapitre 7: Measure de Haar. Chapitre 8: Convolution et Représentations; Actualites Scientifiques et Industrielles 1306; Hermann: Paris, France, 1963.

7. Protasov, I.V. Dualisms of topological abelian groups. Ukr. Math. J. 1979, 31, 207-211. [CrossRef]

8. Cornulier, Y. On the Chabauty space of locally compact abelian group. Algebr. Geom. Topol. 2011, 11, 2007-2035. [CrossRef]

9. Protasov, I.V. On the Souslin number of the space of subgroups of a locally compact group. Ukr. Math. J. 1988, 40, 654-658. [CrossRef]

10. Montgomery, D.; Yang, C.T. The existence of a slice. Ann. Math. 1957, 65, 108-116. [CrossRef]

11. Bredon, G.E. Introduction to Compact Transformation Groups; Academic Press: New York, NY, USA; London, UK, 1972.

12. Tsybenko, Y.V. Dyadic spaces of subgroups of a topological group.Ukr. Math. J. 1986, 38, 635-639.

13. Pourezza, I.; Hubbard, J. The space of closed subgroup of $\mathbb{R}^{2}$. Topology 1979, 18, 143-146. [CrossRef]

14. Kloeckner, B. The space of closed subgroups of $\mathbb{R}^{n}$ is stratified and simply connected. J. Topol. 2009, 2, 570-588. [CrossRef]

15. Protasov, I.V.; Tsybenko, Y.V. Connectedness in the space of subgroups. Ukr. Math. J. 1983, 35, 382-385. [CrossRef]

16. Leiderman, A.; Protasov, I.V. Cellularity of a space of subgroups of a discrete group. Comment. Math. Univ. C 2008, 49, 519-522. 
17. Protasov, I.V.; Tsybenko, Y.V. Chabauty topology in the lattice of closed subgroups. Ukr. Math. J. 1984, 36, 207-213. [CrossRef]

18. Mazurov, V.D.; Khukhro, E.I. (Eds.) Unsolved Problems in Group Theory; 13th ed.; Russian Academy of Sciences Siberian Division, Institute of Mathematics: Novosibirsk, Russia, 1995.

19. Protasov, I.V. Closed invariant subgroups of locally compact groups. Dokl. Acad. Nauk SSSR 1978, 239, 1060-1062.

20. Protasov, I.V. Local theorems for topological groups. Izv. Acad. Nauk SSSR. Ser. Mat. 1979, 43, 1430-1440. [CrossRef]

21. Mal'tsev, A.I. Selected Works. Classic Algebra; Nauka: Moscow, Russia, 1976; Volume 1.

22. Protasov, I.V. Local method and compactness theorem for topological algebraic systems. Sib. Math. J. 1982, 23, 136-143. [CrossRef]

23. Grigorchuk, R.I. Degrees of growth of finitely generated groups and the theory of invariant means. Math. USSR Izv. 1985, 25, 259-330. [CrossRef]

24. Gromov, M. Groups of polynomial growth and expanding maps. Inst. Hautes Etudes Sci. Publ. Math. 1981, 53, 53-73. [CrossRef]

25. Cornulier, Y.; Guyot, L.; Pitsch, W. On the isolated points in the space of groups. J. Algebra 2007, 307, $254-277$. [CrossRef]

26. Klyachko, A.A.; Olshanskii, A.Y.; Osin, D.V. On topologizable and non topologizable groups. Topol. Appl. 2013, 160, 2014-2020. [CrossRef]

27. Glasner, E.; Weiss, B. Uniformly recurrent subgroups. In Recent Trends in Ergodic Theory and Dynamical Systems (Contemporary Mathematics, 631). AMS, Providence; American Mathematical Society: Providence, RI, USA, 2015; pp. 63-75.

28. Matte Bon, N.; Tsankov, T. Realizing uniformly recurrent subgroups. arXiv 2017, arXiv:1702.07101.

29. Protasov, I.V. Topological dualizms of locally compact abelian groups. Ukr. Math. J. 1977, 29, 625-631.

30. Protasov, I.V. Topological groups with compact lattice of closed subgroups. Sib. Math. J. 1979, 20, 378-385. [CrossRef]

31. Protasov, I.V. 0-dimensional groups with compact space of subgroups. Math. Zamet. 1985, 37, 483-490. [CrossRef]

32. Protasov, I.V. Compacts in the space of subgroups of a topological groups. Ukr. Math. J. 1986, 38, 600-605. [CrossRef]

33. Protasov, I.V. Topological groups with $\sigma$-compact spaces of subgroups. Ukr. Math. J. 1985, 37, 93-98. [CrossRef]

34. Protasov, I.V.; Saryev, A. Topological abelian groups with locally compact lattice of closed subgroups. Dopov. AN Ukrain. SSR. Ser. A 1980, N3, 29-32.

35. Protasov, I.V. Limits of compact subgroups of a topological groups. Dopov. AN Ukrain. SSR. Ser. A 1986, N5, 64-66.

36. Panasyuk, S.P. Metrizability in the space of subgroups of a Lie group. Ukr. Math. J. 1990, 42, 351-355. [CrossRef]

37. Panasyuk, S.P. Normality in the space of subgroups of a Lie group. Ukr. Math. J. 1990, 42, 786-788. [CrossRef]

38. Piskunov, A.G. Reconstruction of the Vectoris topology by compacts in the space of closed subgroups. Ukr. Math. J. 1990, 42, 789-794. [CrossRef]

39. Bourbaki, N. Éléments de Matématique. Fascicule II. Livre III: Topologie Générale. Chapitre 1: Structures Topologiques. Chapitre 2: Structures Uniformes; Hermann: Paris, France, 1940.

40. Protasov, I.V.; Saryev A. Bourbaki spaces of topological groups. Ukr. Math. J. 1990, 42, 542-549 [CrossRef]

41. Romaguera, S.; Sanchis, M. Completeness of hyperspaces of topological groups. J. Pure Appl. Algebr. 2000, 149, 287-293. [CrossRef]

42. Protasov, I.V.; Saryev A. Semigroup of closed subsets of a topological group, Izv. Akad. Nauk TadzhSSR. Ser. Fiz.-Tekh. Nauk 1988, 3, 21-25.

43. Itzkowitz, G.L. Continuous measures, Baire category, and uniformly continuous functions in topological groups. Pac. J. Math. 1974, 54, 115-125. [CrossRef] 
44. Protasov, I.V. Functionally balanced groups. Math. Acad. Sci. USSR 1991, 49, 87-90. [CrossRef]

45. Protasov, I.V. Order convergence in the lattice of subgroups of a topological group. Izv. Vyšs. Učebn. Zav. Matematika 1980, 9, 25-29.

46. Scheiderer, C. Topologies on subgroup lattice of a compact group. Topol. Appl. 1986, 23, 183-191. [CrossRef]

47. Protasov, I.V.; Stukotilov, V.S. Ochan topologies on a space of closed subgroups. Ukr. Math. J. 1989, 41, 1337-1342. [CrossRef]

48. Protasov, I.V. On topologies on lattices of subgroups. Dopov. AN Ukr. SSR Ser. A 1981, N11, $29-32$.

49. Roe, J. Lectures on Coarse Geometry; AMS University Lecture Ser. No. 31; American Mathematical Society: Providence, RI, USA, 2003.

50. Protasov, I.V.; Zarichnyi, M. General Asymptopogy; Mathematical Studies Monograph Series 12; VNTL: Lviv, Ukraine, 2007.

51. Dikranjan, D.; Protasov, I.; Zava, N. Hyperballeans of groups. Topol. Appl. 2018, in press.

(C) 2018 by the author. Licensee MDPI, Basel, Switzerland. This article is an open access article distributed under the terms and conditions of the Creative Commons Attribution (CC BY) license (http:/ / creativecommons.org/licenses/by/4.0/). 